\title{
Mechanism of High-Speed-Waterdrop Erosion of Methyl Methacrylate Plastic
}

\author{
Olive G. Engel
}

\begin{abstract}
A mechanism for the high-speed-waterdrop erosion of methyl methacrylate plastic is postulated as a result of a parallel study of the damage that is done by the impacts of steel spheres and of deforming lead pellets. Results of the parallel study are described. The mechanism can be extrapolated with safety only to other brittle materials of low tensile strength, the other characteristic properties of which are closely similar to those of methyl methacrylate plastic.
\end{abstract}

\section{Introduction}

The erosion that waterdrops cause when they strike solid surfaces at very high velocities has recently received considerable attention because of the damage that is produced when high-speed aircraft fly through rain. This erosion is essentially the same as that which occurs in steam turbines because of the waterdrops in wet steam. The process has been studied for the past 25 years [1], ${ }^{1}$ but no clear understanding of the complete damage mechanism has emerged.

Any attempt to explain the damage that results from high-speed-waterdrop impingement must take into account the properties of the waterdrop under impact conditions because the high-speed waterdrop is the damage tool. That is, the destructive force that causes this type of damage is a result of the impact of the waterdrop against the solid surface. Under impact at a velocity of the order of the speed of sound in air, water acts as though it were hard, but it retains its liquid property of flow. It has recently been estimated [2] that the impact pressure that results from a collision of this type at an impact velocity of $600 \mathrm{mph}$ is of the order of $30,000 \mathrm{psi}$ and that the radial-flow velocity is close to 1,400 mph.

The damage that results from these high-speed liquid-to-solid collisions is equally a function of the properties of the structural material that sustains the damage. The response of materials of different properties will be different enough to introduce notable modifications in the erosion process.

The analysis of a difficult problem may sometimes be accomplished by reducing it to a simpler problem that is easier to understand. It was suggested by Schröter [3] in regard to cavitation erosion, for example, that physically parallel studies might be more rewarding than further observations of the cavitation progress itself because the cavitation cannot be reduced to slow motion nor separated from other phenomena. As just pointed out, under impact conditions a waterdrop behaves like a hard sphere, but unlike a sphere of hard, solid material, it retains its. liquid property of flow. The results of the parallel study of the impact of steel spheres, and especially of deforming lead pellets, on methyl

Figures in brackets indicate references at the end of this paper. methacrylate plastic appears to indicate the mechanism of high-speed-rain erosion.

\section{Collision of Steel Spheres and of Deform- ing Lead Pellets with Methyl Methacrylate Plastic}

The plastic blocks used for this study were of Plexiglas I-A obtained from the Rohm \& Haas Co. in $1 / 4^{-}, 1-$, and 3 -in. thicknesses. The projectiles were $7 / 32$-in. steel spheres, 0.22 -in.-diameter soft deforming lead pellets that were shot with small charges of powder from a 0.22 -caliber gun, and $5 / 16$-in. steel spheres that were shot from a 0.30 -caliber gun. The impact velocities were determined by momentum transfer to a pendulum through the block of plastic.

The impacts resulted in a characteristic type of fracture of the plastic. At relatively low velocities of impact, circles of short, very fine, straight cracks were observed (see figs. 1, 2, and 3). These straight cracks were perpendicular to radii from the central point of impact. The center spot of the impact was, however, completely without any such cracks. At relatively high velocities of impact, the circular region of fine cracking on the impact surface was bounded by a very well-defined circular crack when the projectiles were steel spheres. Such a crack was not observed at these velocities when the projectiles were lead pellets. However, in the latter case, a circular subsurface shear crack was produced. These subsurface shear cracks appear as large dark circles, with their center at the center of the damage site in figure 3. They are out of focus with the surface details.

Radial cracking resulted from the impacts of steel spheres but not from those of deforming lead pellets. On thin plates of Plexiglas I-A and at low velocities the radial cracking appeared first on the side of the plate opposite to the impact face. At high velocities on these thin plates the radial cracks penetrated through the plate to the impact surface. On the thick plates, very short radial cracks appeared on the impact surface itself. They were observed only at relatively high velocities.

A detailed discussion of the specific types of cracking and of a model that explains the observations is contained in the following sections. 


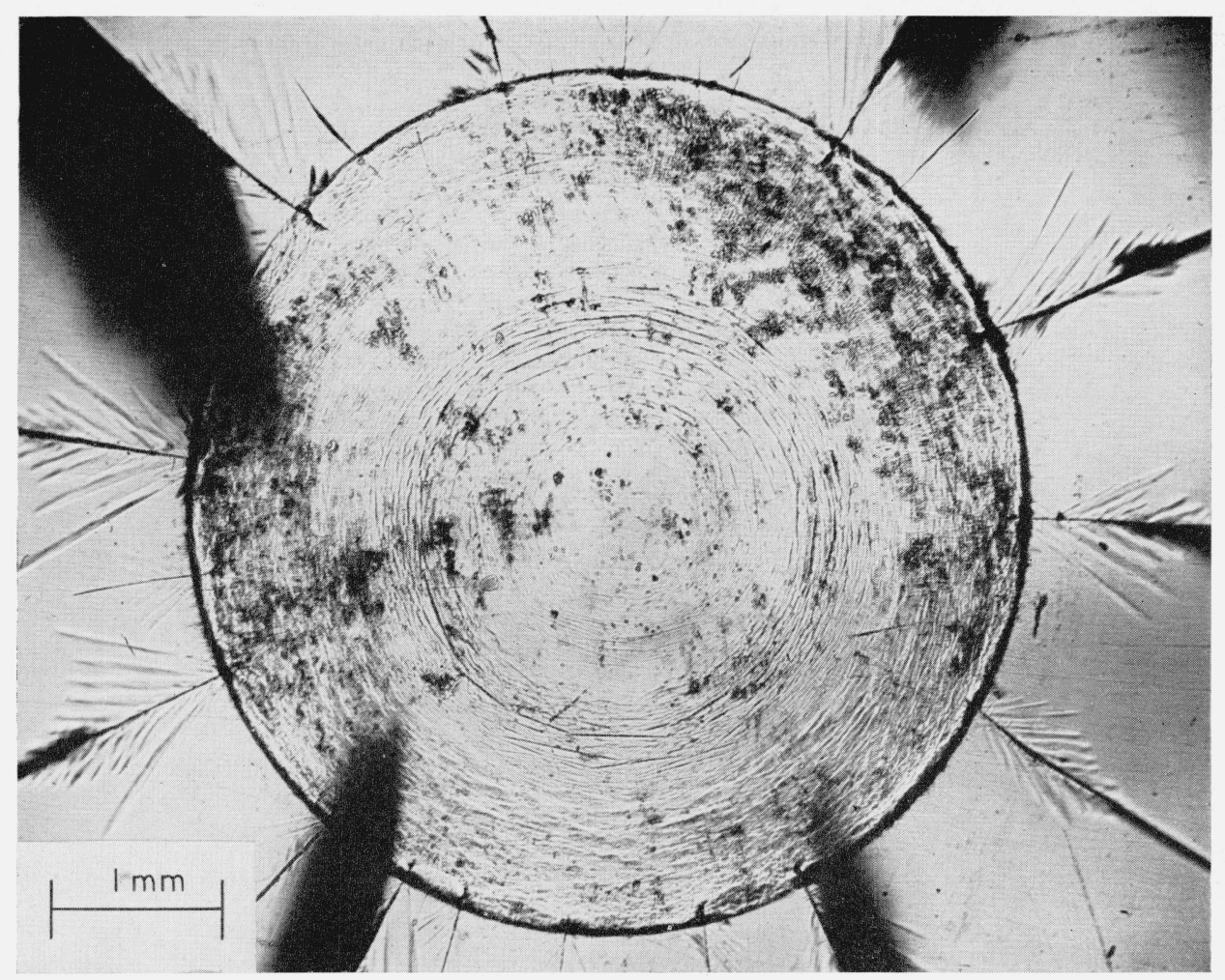

FIGURE 1. Damage site produced by a steel sphere on impinging against Plexiglas $I-A$.

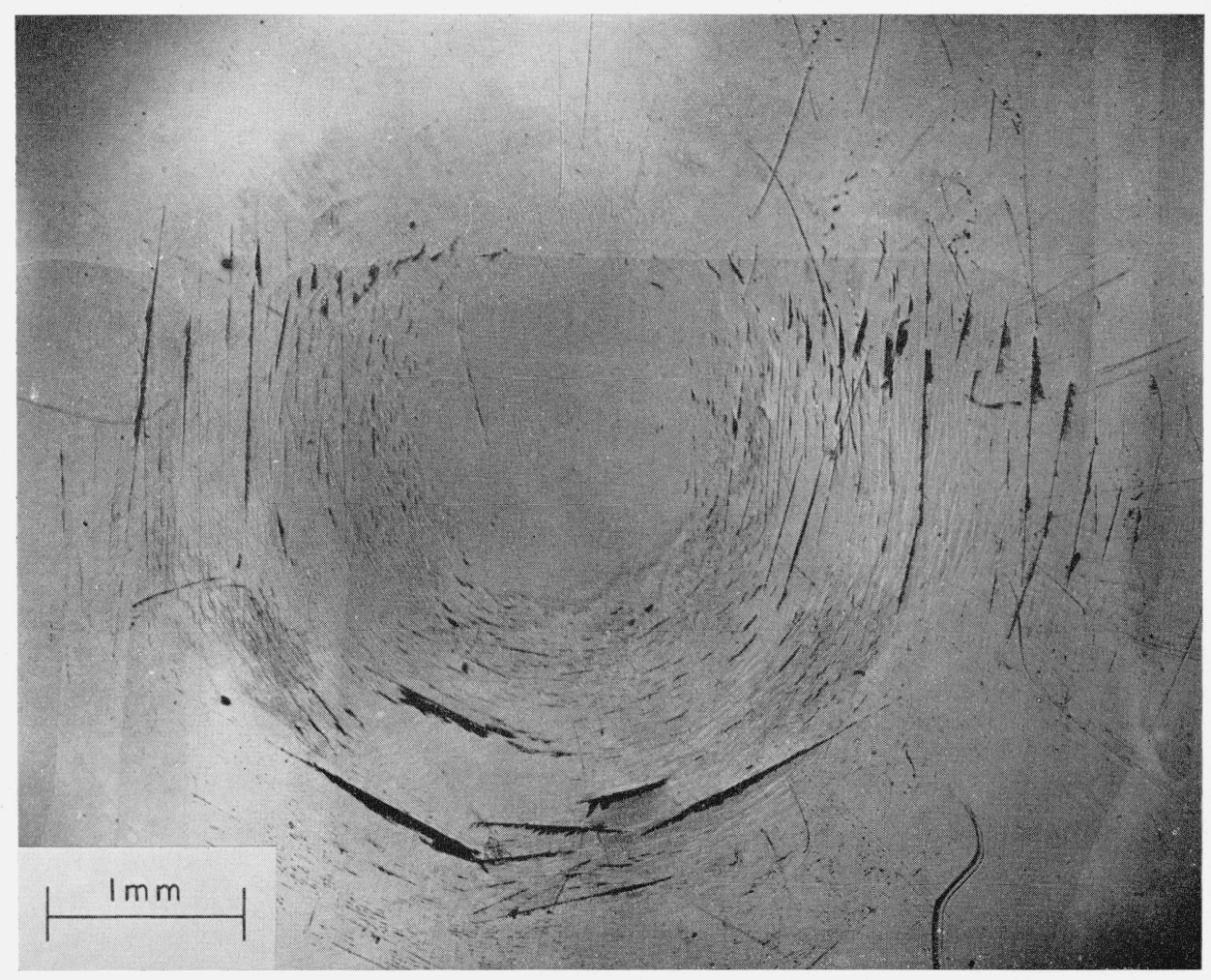

FIGURE 2. Damage site produced by a deforming lead pellet on impinging against Plexiglas $I-A$. 


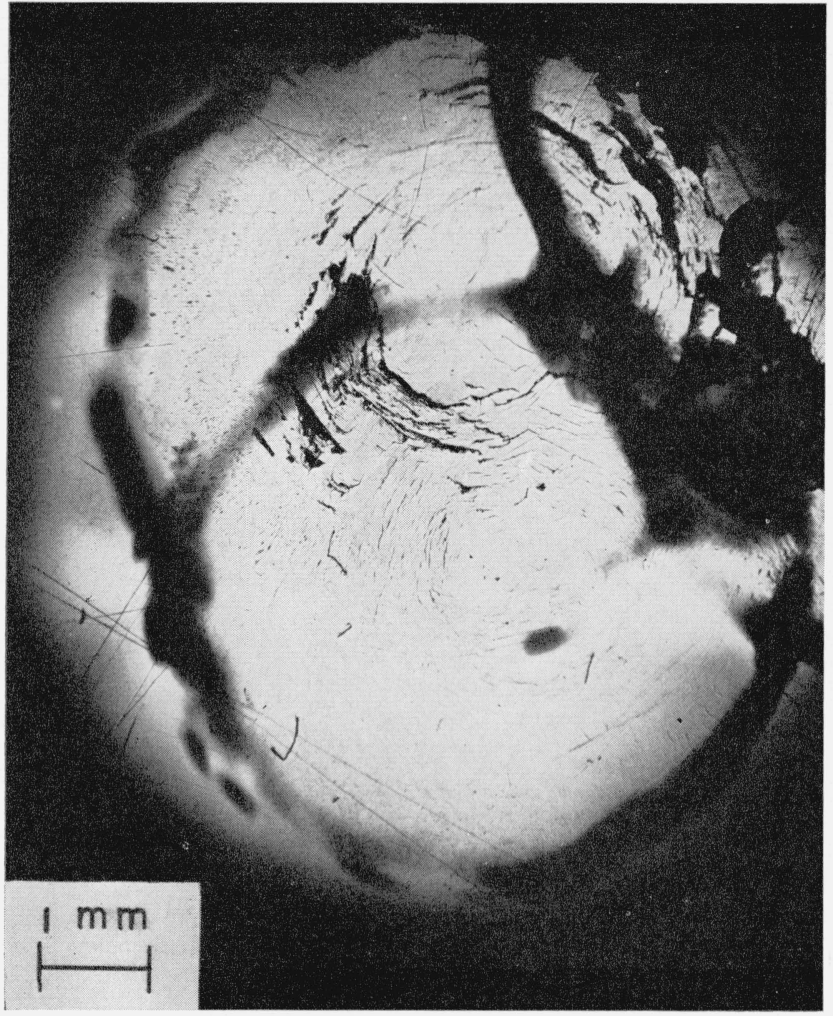

Figure 3. Damage site produced on Plexiglas $I-A$ as a result of a multiple shot with deforming lead pellets.

The center area of two shots can be seen in the picture. Part of the damage caused by two other shots can be seen to one side.

\subsection{Circular Crack and Fine Cracking in the Circular Zone}

At low impact velocities both for impacts of steel spheres and for impacts of deforming lead pellets the damage consisted only of a few circles of fine, short, straight cracks that enclosed the central point of the impact. The central point of the impact itself was free of any cracking (see figs. 1, 2, and 3). As the velocity was increased, the amount of fine cracking increased, and the diameter of the zone of fine crack ing increased. Finally, a velocity was reached at which a well-defined crack formed around the circular zone of fine cracking when steel spheres were used as projectiles (see fig. 1). This outer crack was too deep for a depth measurement with the interferometer. When deforming lead pellets were used, subsurface shear cracks formed at high velocities. The subsurface shear cracks appear as broadened arcs of a circle out of focus with the surface details (see fig. 3).

The diameter of the circular zone of fine cracking for comparable velocities was influenced by the thickness of the plastic plate only in the case where the plate thickness was as low as $1 / 4$ in. Comparison of the diameters of damage sites on 1-in.-thick and on 3-in.-thick blocks of Plexiglas I-A for 5/16-in. spheres at comparable velocities showed that they

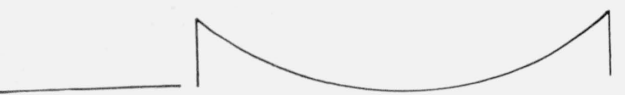

FIGURE 4. Cross section of a region of fine cracking bounded by a well-defined circular crack similar to that shown in figure 1

were essentially the same. Comparison of the diameters of damage sites on 1/4-in.-thick and on 3-in.thick Plexiglas I-A for 516 -in. spheres at comparable velocities showed that the diameters on the $1 / 4$-in.thick material were larger.

The diameter of the circular zone of fine cracking for comparable velocities also varied with the diameter of the steel sphere. Comparison of the diameter of the damage site produced by a $7 / 32$-in. sphere with that produced by a $5 / 16$-in. sphere on $1 / 4$-in.-thick Plexiglas I-A at the same velocity showed that the damage site produced by the larger sphere was the larger.

An interferometric study of the circular region bounded by fine cracking indicated that the cross section of this region must be as indicated in figure 4 .

When deforming lead pellets were used, the cracks were widened and material was broken out of the surface. The widening of the cracks was in the direction of the radial flow of the lead. The breaking out of material was along cracks, was in the direction of the flow of the lead, and was on the side of the crack away from the center of the impact (see figs. 2 and 3). The widening of cracks and the breaking out of material was notably absent where steel spheres were used as projectiles.

\subsection{Radial Cracking}

Cracks appeared that went out radially from the circular crack in the case of the impacts of steel spheres. They were especially evident in the damage marks left by the impact of steel spheres against the thick blocks of plastic. These cracks extended only a very short distance within the circular region of fine cracking. In figure 1 they appear as featherlike structures around the circular crack.

There was also another type of cracking that occurred as a result of the impact of steel spheres when the plastic plates were thin. This was a radial, or star-shaped, cracking similar to that which results from the impact of a small, hard object against thin glass plates. In the case of $1 / 4$-in.-thick Plexiglas I-A these radial cracks appeared on the side of the plastic opposite the impact face at relatively low velocities. They converged under the central point of the collision. They did not extend all the way through the plate to the impact surface. Furthermore, there was no circular crack or circular area of fine cracking on this reverse face of the plate. At high velocities these radial cracks extended completely through the plate to the impact surface.

These radial cracks were quite long. In the case of impacts of $5 / 16$-in. spheres at the relatively low velocities of 232 and $269 \mathrm{ft} / \mathrm{sec}$ the cracks were over $2 \mathrm{in}$. long and for the higher of these two velocities 
were close to 3 in. long. On the other hand, radial cracks that resulted from the impact of $7 / 32$-in. spheres at the high velocities of 611 and $943 \mathrm{ft} / \mathrm{sec}$ were only about $1 \frac{1}{2}$ in. long. From this it would appear that the length of these cracks may be a function both of velocity and of sphere diameter. It would seem that the total crack length should correlate with the energy transferred to the plastic by the sphere.

Bowman, Smith, and Kies [4] have plotted total length of radial cracking against velocity and have found a maximum in the curve for Plexiglas I-A at a velocity of $775 \mathrm{ft} / \mathrm{sec}$, and for Plexiglas II at a velocity of about $875 \mathrm{ft} / \mathrm{sec}$. They were using $5 / 16$-in.-diam steel spheres. The shots were made against 6 -in.-square plates of plastic that were $\frac{1 / 4}{4}$ in. thick. The reported maximum is a suppressed maximum that was observed under these experimental conditions. They found, furthermore, that Plexiglas II that had been subjected to hot-work reached maximum crack length at a lower velocity than Plexiglas II in the as-received condition.

\subsection{Crazing in a Circle}

The fine cracking that has been observed at the damage sites produced by the impacts of steel spheres and of deforming lead pellets on Plexiglas I-A may be designated as crazing. In the case of the cup-shaped depression that must form when a steel sphere or lead pellet strikes a plastic surface, the tensile stresses extend as radii from the central point of impact, which is under compression, to the rim of the cup-shaped cavity. The fine cracking that forms as a result of these tensile stresses is more or less parallel in tiers that form at an angle to one another, but the individual fine cracks are not mutually parallel.

Recently, Wolock and Newman [5] have traced the cross section of a craze crack of the generally accepted type with the interferometer. They have found that the surface material is raised on both sides of the crack (see fig. 5). It may be postulated that such cracks form as stress relievers after the material has reached the limit of stress relief from plastic flow, or if plastic flow cannot take place fast enough. If plastic flow has occurred either prior to or concomitant with the formation of the craze crack, the material will tend to come back into its original position after the stress is relieved, and the crack will be raised on each side as is observed. As a part of the present investigation, Dr. Newman made a similar interferometric study of the fine cracking that was produced by the impact of a steel sphere on Plexiglas $\mathrm{I}-\mathrm{A}$ and found that a raised ridge exists on both sides of the crack, as shown in figure 5 .

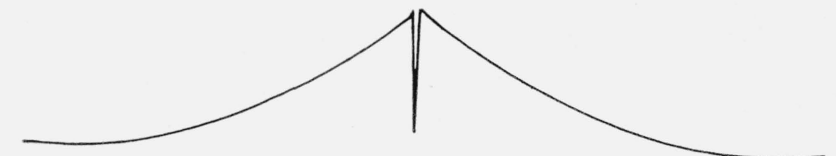

FIgure 5. Cross section of a craze crack (or fine crack) as found by use of the interferometer.
2.4. A Model for the Impact Damage of Deforming Lead Pellets and Steel Spheres on Methyl Methacrylate Plastic

The observations that have been described can be explained rather easily by a simple model in which it is assumed that the impact of a steel sphere or of a deforming lead pellet produces a cup-shaped cavity in the plastic by compressing it. Tensile stresses should then be introduced in the regions indicated as $\mathrm{A}, \mathrm{B}$, and $\mathrm{C}$ in figure 6 . The stresses in region $\mathrm{A}$ should become important as soon as any depression takes place, regardless of how shallow it may be. On the other hand, stresses that are introduced in the regions indicated as B and $\mathrm{C}$ may only become appreciable after the cup-shaped cavity reaches the more acute stage of depression indicated schematically in figure 6 . The stresses in region B exist over the relatively sharp angle between the undisturbed surface and the wall of the depression.

It is postulated in section 2.3 that craze cracks may form as stress relievers after a material that is under tensile stress has reached the limit of stress relief from plastic flow or if plastic flow cannot take place fast enough. Similarly, the fine cracking, which appears to be crazing, should serve to relieve the tensile stresses, indicated as $\mathrm{A}$ in figure 6 , that are produced as the plastic material is depressed by the impact. A cavity as deep as that indicated schematically in figure 6 will only be produced at high impact velocities and only by a sphere that does not flow on impact. Hence, the tensile stresses at B should only become acute at high velocities, and then only in the case where a steel sphere rather than a deforming lead pellet is the projectile. These stresses may be responsible for the well-defined circular crack that forms at high impact velocities around the circular region of fine cracking in the damage sites produced by the impact of steel spheres. The tensile stresses, indicated as $\mathrm{C}$ in figure 6 , may be responsible for the feather-like cracks that go out

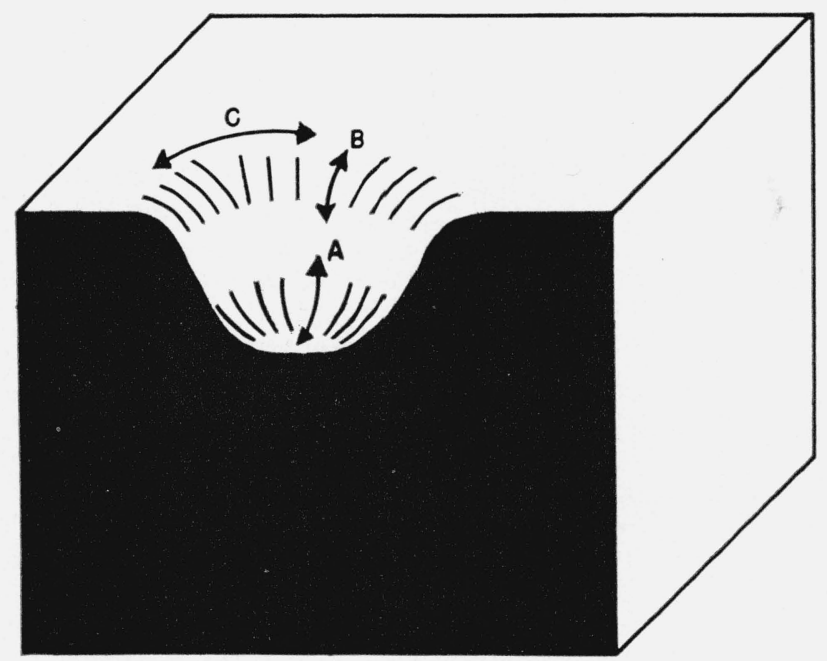

FigurE 6. Tensile stresses at a cavity formed by pressing a hard sphere into deformable material. 
radially from the circular crack in the case of the impacts of steel spheres.

On this model, figure 6 , there should be severe compression during the impact where the hard sphere is bearing against the plastic to form the cupshaped cavity. McAdam, Geil, and Jenkins [6] have pointed out that material under compression does not tend to undergo a tensile failure. It is notable that there is no fine cracking in the severely compressed region at the center of the damage sites (see figs. 1, 2, and 3).

In the light of the model that has been assumed, essentially the same diameter of circular cracking would be expected to occur on a 1 -in.-thick plate as on a 3-in.-thick plate of plastic, that is, for those plate thicknesses that are large in comparison with the shot diameter. It is also expected that for a much thinner plate, the diameter of the damage site would be increased by the bowing of the plate as a whole around the impinging sphere or pellet. This was observed to be the case.

Furthermore, on the model that has been assumed, a gradient of tensile stress would be expected up the sides of the cup-shaped depression, that is, along the arrow marked $A$ in figure 6 . Griffith [7] derived an expression that showed that there should be a maximum crack length for every value of stress that produced a crack. A magnification of the finecracked circular region produced by one of the shots shows some evidence that the lengths of the fine cracks at any given distance from the central point of impact are, in general, qualitatively of the same size, and that there is a size gradient from the rim to the bottom of the depression. However, there are many exceptions to this observation.

The radial cracks that are observed when the projectiles are steel spheres and that appear first on the reverse side of the plate can be explained as tensile failures. After the compressional wave produced by the impact on a relatively thin plate (onedimensional case) has just moved through the plastic, the plastic material on the free face opposite the impact surface should tend to bulge when the compressional wave reflects there as a tensile wave. It is possible that a star-shaped hole may open to relieve the tensile stress. After the compressional wave has reflected as a tensile wave, the plastic material should snap back into a star consisting of radial cracks only. A search was made for this effect by H. L. Smith [8], using a high-speed camera operating at 15,000 frames a second. The starshaped crack appeared, however, in the space of one frame. It is possible that the stress may be relieved sufficiently by crack formation alone, and that a star-shaped hole may never form. A camera having a higher operating speed will be needed to determine this.

The deforming lead pellets used as projectiles flowed on impact to approximately twice their original diameter. This flow of the lead produces a force against any surface irregularity in its path which, in conjunction with the restraint of the irregularity by the underlying material, results in a torque tending to break the material in the irregularity away from the underlying material. The raised ridges along the fine cracks could constitute bearing points for this torque and offer an explanation for the observed fact that where lead pellets were used as projectiles the cracks were widened and material was broken out of the surface along the cracks and in a direction away from the center of the impact (the direction of the flow of the lead) (see figs. 2 and 3).

This flow property of the lead also explains the subsurface shear cracks that appear as large dark circles out of focus with the surface details in figure 3 . The flow of the lead over the surface while it is simultaneously compressed against the surface would tend to move the surface layer of the plastic with respect to the under layers. The subsurface cracks are not observed in the damage sites produced by the steel spheres, which did not flow as a result of the collision.

It is the flow property of the deforming lead pellets that makes the damage sites produced by them different from the damage sites produced by the steel spheres. The fracture that results from the impact of deforming lead pellets is more nearly related to what must happen when liquid drops strike a surface than is the fracture that results from the impact of steel spheres. In the case of waterdrops, of course, the damage from a single blow cannot be as decisive as that which results from a single impact of a deforming lead pellet because the density of water is so much lower than that of lead. The damage caused by high-speed impact of waterdrops on methyl methacrylate plastic is discussed in detail in the following sections.

\section{High-Speed-Rain Erosion of Methyl Meth- acrylate Plastic}

The preceding study of the impact of steel spheres and of deforming lead pellets on methyl methacrylate plastic is a physically parallel study to the highspeed-rain erosion of brittle plastics of low tensile strength. To make a realistic approach to the mechanism of the high-speed-rain erosion process, it is essential to keep in mind that the projectile is a water sphere that acts as though it were hard but that is capable of an extremely fast radial flow the instant after it impinges.

The process of high-speed-rain erosion should be very similar to the process of producing damage by impacts with deforming lead pellets. For plastics that are similar in properties to methyl methacrylate plastic, radial fine cracking should form around the point of impact of a hard, impinging, spherical waterdrop due to the tensile stresses that are produced when the compressive impact stress forms a cupshaped cavity in the material. A breaking out of material from the surface should be observed as a result of the rapid radial flow of the water after the collision over the raised ridges of the fine cracks that have formed. 




FigurE 7. Circles of fine rracks produced on Lucite by high-speed-waterdrop impacts.

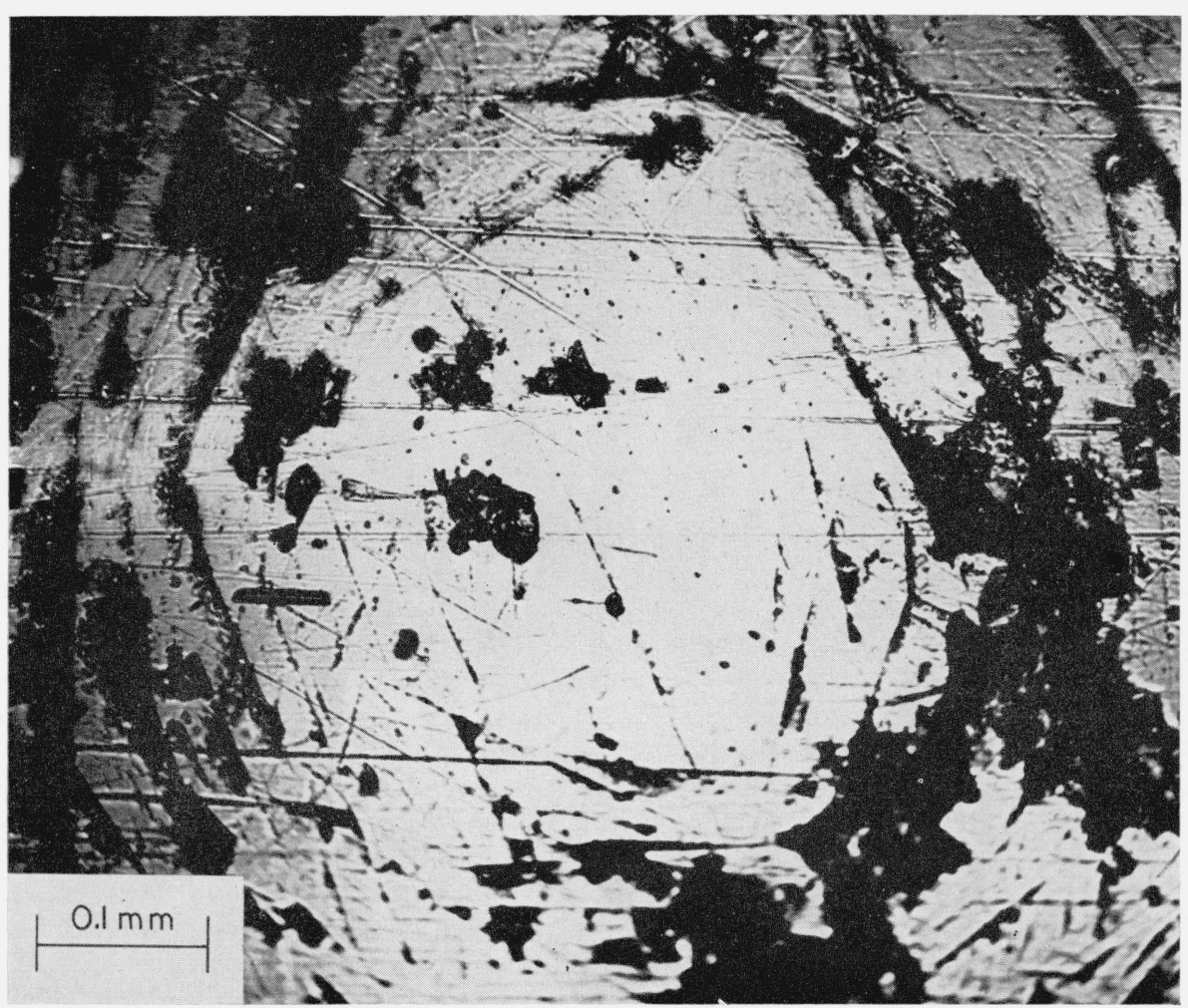

FiguRE 8. Magnified view of the most prominent circle of fine cracks shown in figure 7 . 


\subsection{Circular Fine Cracking as a Result of High- Speed-Water Blows}

A search for damage sites comparable to those that are observed as a result of impacts of deforming lead pellets was at first unrewarded. Specimens of methyl methacrylate plastic that were rotated at a velocity of $500 \mathrm{mph}$ through 1 -in. $\mathrm{hr}$ artificial rain at the Cornell Aeronautical Laboratory for periods of 30 to $40 \mathrm{sec}$ were already in an advanced stage of erosion. The first damage sites were obscured by later overlapping impacts, so that nothing could be concluded in regard to their initial configuration. No general pattern was visible, and all that could be concluded from them was that the erosion damage went out from cracks and crack intersections that may or may not have been there before the water struck.

However, individual damage sites could be seen on a specimen similarly exposed for only $25 \mathrm{sec}$. Figure 7 shows the result. The configurations that stand out in the photograph are the circles of fine cracking. Here again, the circles of parallel lines in tiers can be seen. It is also notable that here again the craze cracks meet at an angle. In figure 7 the widening of these cracks due to the outward flow of the water from the central point of impact can be seen. Direct comparison should be made with figures 2 and 3, where the same configuration of lines and the same widening of cracks can be seen as the result of the impact and flow of deforming lead pellets.

Figure 8 is a higher magnification of the most prominent circle shown in figure 7 . In figure 8 the angular meeting of the parallel lines is apparent as is also the widening of the cracks by breaking out of the surface material along them in a direction away from the central point of impact as a result of the radial flow of the water. From figures 2, 3, 7, and 8 the conclusion can be drawn that the mechanisms of damage produced by the impact of deforming lead pellets and by the high-speed impingement of waterdrops on methyl methacrylate plastic are identical. The difference between them is one of degree only.

\subsection{Comparison of the Destructive Action of the Impact of Lead Pellets and the Impact of Water- drops}

The destructive action of these flowing projectiles is due (A) to the impact of the hard sphere that constitutes the projectile itself, and (B) to the radial flow of the projectile after the collision, as follows:

(A) The first of these two types of damage is the production of a circle of fine cracks. With respect to this type of damage, the impacts of lead pellets are more destructive than the impacts of waterdrops, as can be seen by comparing the amount of fine cracks produced by a single impact in figures 2 and 3 with that produced by a single impact in figure 7 . The impact velocity of the lead pellets that produced the damage sites shown in figure 3 was about half that of the waterdrops that produced the damage sites shown in figure 7 . The lead-pellet velocity that produced the damage sites shown in figure 3 was appreciably higher than that which produced the damage site shown in figure 2. It is easy to see that the extent of damage observed in figures 2 and 7 is a logical result of the kind of flowing projectile that produced it. The impact pressure that results when a spherical liquid projectile strikes a flat, solid surface that has an infinite modulus of elasticity is given by [2]

$$
P=(\alpha / 2) c \rho v_{0}
$$

where $P$ is the impact pressure, $\alpha$ is a coefficient less than unity that arises from the flow property of the liquid projectile, $c$ is the speed of sound in the liquid projectile, $\rho$ is the density of the liquid projectile, and $v_{0}$ is the impact velocity. Lead has a much higher density than water and a value of $\alpha$ that is closer to unity. Therefore, according to eq (1), a lead pellet should exert a greater impact stress than a water sphere. The lead pellet should form a deeper depression than the waterdrop does in the surface of the plastic as a result of its collision, should produce more tensile stress in the surface about the depression that it produces, and consequently should produce more fine cracking.

(B) The second type of damage is (a) the widening of the surface cracks and the breaking out of material from the surface, both of which are observed in the damage sites produced by waterdrops as well as in those produced by lead pellets, and (b) the subsurface shear cracks that are observed only in the damage sites produced by the lead pellets. The damage (a) may result from the application of the external torque exerted by the rapid radial flow along the surface against irregularities in the surface. In this connection the raised ridges of the craze, or fine cracks, may be a factor. The damage (b) appears to be due to a shear stress that tends to move the surface layer of the plastic solid with respect to the underlying layers.

Whether the damage caused by the flow as a result of impact of a waterdrop or of a lead pellet is considered more severe depends on the criterion that is taken for the extent of damage. The waterdrop flows out to many times its original diameter and therefore breaks a large amount of material from the surface. The lead pellet flows only to about twice its original diameter and hence is less effective than water in this respect. On the other hand, the shear stress exerted by the flow of the lead is much greater than that exerted by the flow of the water, because it produces subsurface shear cracks, which the water does not produce even when its impact velocity is about double that of the lead. On the basis of its shear stress, therefore, the radial flow of the lead is more destructive than that of the water. 


\subsection{Shear Stress Due to the Radial Flow of an Impinging Waterdrop}

The shear stress, $\tau$, between layers of liquid in laminar flow is given by the product of the viscosity, $\mu$, and the velocity gradient through the moving sheet of liquid perpendicular to its direction of flow. That is,

$$
\tau=\mu \frac{\partial v}{\partial z}
$$

where $v$ is the velocity of the sheet of liquid, and $z$ is the direction through the thickness of the liquid sheet. The layer of liquid molecules in direct contact with the solid has zero velocity, but the velocity gradient is not zero, and the shear stress is applied to the solid.

Faust [9] and Hyde [10] have found that the viscosity of liquids not only increases with pressure, but it increases at an increasing rate as the pressure rises. Hence, it seems entirely reasonable to conclude that water impacts will produce subsurface shear cracks at sufficiently high impact velocities, even though they are not observed in the views shown in figures 7 and 8 , where the relative impact velocity was $500 \mathrm{mph}$.

It is possible to estimate roughly the shear stress exerted by the radial flow of a waterdrop that strikes a solid surface at a relative velocity of 500 mph. In developing an equation for the impact pressure under an impinging water sphere [2], it was shown that the thickness, $\delta$, of the sheet of water in radial flow when the pressure is at a maximum is given by

$$
\delta=4 r(1-\alpha)^{2}\left(\frac{v_{0}}{c}\right)^{2},
$$

where $r$ is the radius of the flattened water sphere, $\alpha$ is a coefficient that arises from the flow property of the liquid and that is very close to unity at high impact velocities, $v_{0}$ is the relative impact velocity between the water sphere and the surface that it strikes, and $c$ is the speed of sound in water. The median radius of 1 -in./hr raindrops, such as were used in producing the damage sites in figures 7 and 8 , is $0.095 \mathrm{~cm}$, so that the radius of the flattened sphere is $9.5 \mathrm{~cm}$. If the coefficient $\alpha$ is taken to be 0.99 , then $\delta$ is found to be $0.00008 \mathrm{~cm}$.

The average velocity of the radial water flow at the time of maximum pressure also was found [2] to be given by

$$
\ddot{u}=\sqrt{c \alpha v_{0}} .
$$

For a relative impact velocity, $v_{0}$, of $500 \mathrm{mph}$, the radial flow velocity, $v$, is found to be about 58,000 $\mathrm{cm} / \mathrm{sec}$. As this is an average value of the radial flow velocity, a rough estimate of the velocity gradient through the radially moving sheet of water can be made by taking it to be equal to $\Delta v / \Delta z$, where $\Delta z$ is half the value of $\delta$. To this degree of approximation, $\tau$ is found to be $210 \mathrm{psi}$, because the viscosity of water at room temperature is about 0.01 poise. This value is too low because of the increase of the viscosity with pressure. From the data of Faust [9] the viscosity of ethyl alcohol increases by a factor of about three for an increase of pressure comparable to the impact pressure that is produced when a waterdrop strikes a solid surface at a relative velocity of $500 \mathrm{mph}$. If the increase in the viscosity of water, which is also an associated liquid, is about the same as that of ethyl alcohol, then $\tau$ is about $630 \mathrm{psi}$ for the radial flow of a waterdrop that strikes a solid surface at the relative velocity of $500 \mathrm{mph}$. This is below the static shear strength of methyl methacrylate plastic, which is about 8,000 psi [11].

The calculated value of $\tau$ is rather sensitive to the value that is chosen for $\alpha$. The value of 0.99 for $\alpha$ was used in place of the value 0.9 , which has been assumed to be the value of $\alpha$ in previous calculations for this magnitude of the relative impact velocity in order to make the shearing stress as high as possible. It will be interesting to know at what impact velocity subsurface shear cracks will be found to be produced experimentally on methyl methacrylate plastic by water blows. This may come to light when a method to produce higher relative impact velocities between a water sphere and a solid surface is developed.

\section{Mechanism of High-Speed-Rain Erosion on Methyl Methacrylate Plastic}

The effect of high-speed-waterdrop impingement on methyl methacrylate plastic correlates with the damage produced in the parallel study of impacts of steel spheres and especially of the impacts of deforming lead pellets. In the parallel study it was assumed that a cup-shaped cavity is produced in the plastic surface as a result of the compressive load exerted by the impinging projectile. It was further assumed that depressing the surface in this way introduces tensile stresses that, for a brittle material of low tensile strength, produce cracks. In the case of a flowing projectile the cracks are widened, and material is broken out of the surface along the cracks and at crack intersections by the torque that the ultrarapid radial flow exerts.

At high impact velocities a waterdrop behaves like a hard sphere, but unlike a sphere of hard solid material, it retains its liquid property of flow. These properties of the waterdrop under impact conditions make it comparable to the steel sphere and to the deforming lead pellet. It is logical that the mechanism by which it damaged the plastic surface should be entirely comparable to that by which the steel spheres and the deforming lead pellets damaged the surface.

Beal and Wahl [12] have found that for a given relative impact velocity the impingement of waterdrops at a 3 -in./hr rain rate, in which the median drop size is $2.5-\mathrm{mm}$ diam, produces more damage than the impingment of waterdrops at a $1-\mathrm{in}$. hr rain rate, in which the median drop size is $1.9-\mathrm{mm}$ diam. They also found that where three nozzles, each of which produced $1-\mathrm{in} . \mathrm{hr}$ rain with a median drop 
size of $1.9-\mathrm{mm}$ diam, were used, the 3 -in./hr volume of rain was not proportionately more severe than the 1-in./hr volume of rain if the drop size is the same in each. They concluded that the greater damage produced by the 3 -in./hr rain rate with the median drop size of $2.5-\mathrm{mm}$ diam must be due to the larger drop size rather than to the increased number of drops per unit time. In the light of the mechanism that has been postulated, it would follow that this should be the case Comparison of the diameters of damage sites produced by a $7 / 32-i n$. and by a $5 / 16-i n$. steel sphere at the same relative impact velocity and on methyl methacrylate plastic of the same thickness showed that the damage site produced by the larger sphere was the larger (see section 2.1). Similarly, the damage site produced by the average 3 -in. hr raindrop will be larger than the damage site produced by the average 1-in./hr raindrop. The 3-in./hr raindrop will also provide a larger volume of water to produce damage in the process of radial flow. It is notable that eq (1) for the pressure produced by the impact of a water sphere against a flat surface is not a function of the drop size.

Although it would appear that the rain-erosion mechanism on methyl methacrylate plastic has been explained by this study, one cannot safely extrapolate the result to all other brittle plastics of low tensile strength. As pointed out in section 1, the erosion mechanism is not only a function of the impact properties of the waterdrop, but it is also a function of the specific properties of the solid material against which the waterdrop impinges. The mechanism that has been postulated for methyl methacrylate plastic can be extrapolated with safety only to other brittle plastics of low tensile strength having other characteristic properties that are comparable to those of this plastic. Furth and Born [13] have advanced the concept that breaking is a form of melting: "melting being nothing else than a breaking due to the action of the heat movement of the atoms; or putting it the other way round, breaking is nothing else than melting enforced by the action of the stress" (see also references $[14,15,16]$ ). If this concept is correct, a brittle plastic of low tensile strength that differs appreciably from methyl methacrylate plastic either in heat conductivity or in actual melting temperature could respond in a very different manner to the high-speed-water blows.

The author thanks George Irwin of the Naval Research Laboratory for suggesting the parallel study, and Herschel L. Smith and Mark Bowman of the Naval Research Laboratory for making the shots with steel spheres and deforming lead pellets on Plexiglas I-A.

The work described in this paper was sponsored by the Wright Air Development Center. The results that are given were taken from a report submitted to that agency. Their assistance and interest are gratefully acknowledged.

\section{References}

[1] Olive G. Engel, Wright Air Development Center Technical Report 53-192, Part 2 (August 1953).

[2] Olive G. Engel, Wright Air Development Center Technical Report 52-192, Part 1 (July 1953).

[3] H. Schröter, Z. Ver. Deut. Ing. 80, 479 (1936).

[4] Mark Bowman, H. L. Sinith, and J. A. Kies, N.R.L. Mem. Report 181 (June 1953).

[5] S. B. Newman and Irvin Wolock, (publication pending).

[6] D. J. MeAdam, Jr., G. W. Geil, and W. D. Jenkins, Proc. ASTM 4\%, $554(1947)$.

[7] A. A. Griffith, Trans. Roy. Soc. (London) 221 [A] 163 $(1920-21)$.

[8] H. L. Smith (publication pending).

[9] O. Faust, Z. physik. Chem. 86, 479 (1914).

[10] J. H. Hyde, Proc. Roy. Soc. (London) 9z [A] 240 (1920).

[11] Plexiglas Handbook for Aircraft Engineers (Rohm \& Haas Co., 1951)

[12| John L. Beal and Norman E. Wahl, Air Force Technical Report 6190 (1951).

[13| R. Furth and M. Born, Nature 145, 741 (1940).

[14] M. Born, J. Chem. Phys. 7, 591 (1939).

[15] R. Furth, Proc. Roy. Soc. (London) 178 [A] 217 (1941).

[16] R. Furth, Proc. Cambritge Phil. Soc. 37, 34 (1941).

Washington, August 31, 1954. 